\title{
THE SOCIETY'S AWARDS
}

The Society offers a number of valuable awards, most of them annually. Full particulars of the conditions attaching to these awards may be obtained on application to the Secretary.

\section{Society's Gold Medal}

This is the highest honour which the Society can confer for work of an outstanding or fundamental nature in aeronautics.

\section{British Gold Medal for Aeronautics}

The British Gold Medal for Aeronautics is awarded for an achievement leading to advancement in aeronautical science.

\section{Simms Gold Medal}

The Simms Gold Medal is awarded annually for the best paper read in any year before the Society on any science allied to aeronautics, e.g., meteorology, wireless telegraphy, instruments.

\section{The George Taylor (of Australia) Gold Medal}

The Taylor Gold Medal is awarded annually, at the discretion of the Council, for the most valuable paper submitted or read during the previous session.

\section{Wakefield Gold Medal}

The Wakefield Gold Medal is awarded annually to the designer of any invention or apparatus tending towards safety in flying, and is open to members or non-members.

\section{Society's Silver Medal}

The Society's Silver Medal is awarded, at the discretion of the Council, for some advance in aeronautical design.

\section{British Silver Medal for Aeronautics}

The British Silver Medal for Aeronautics is awarded for an achievement leading to advancement in aeronautical science.

\section{Society's Bronze Medal}

The Society's Bronze Medal is awarded, at the discretion of the Council, under the same conditions as those for the Silver Medal, but for some less important advance in aeronautical design.

\section{Wilbur Wright Memorial Premium}

The Wilbur Wright Memorial Lecture is held annually, a premium of seventy-five pounds being awarded to the lecturer invited by the Council to deliver the lecture. The lecture is usually given alternately by an American and an Englishman, and is the most important aeronautical lecture of the year. It is delivered, whenever possible, on the last Thursday in May of each year.

\section{British Commonwealth and Empire Lecture}

The Council of the Royal Aeronautical Society have completed the arrangements for the founding of a British Commonwealth and Empire Lecture.

The lecture, on any aeronautical subject approved by the Council, will be delivered annually in September in London, by a lecturer chosen in alternate years from the British Dominions and Colonies. and Great Britain. 


\section{THE SOCIETY'S AWARDS}

The Council, by founding this British Commonwealth and Empire Lecture, are anxious to encourage new ideas and new points of view from all parts of the British Commonwealth and Empire, and to make the Lecture second only in importance to the Wilbur Wright Memorial Lecture.

The British Commonwealth and Empire Lecture will have a premium of $£ 50$ attached to it, and in the case of lecturers coming from the Dominions and Colonies an allowance will be paid towards the Lecturer's expenses.

\section{R.38 Memorial Prize}

The R.38 Memorial Prize is offered annually for the best paper received by the Society on some subject of a technical nature in the science of aeronautics, preference being given to papers which relate to airships. The prize is twenty-five guineas.

\section{The Herbert Akroyd Stuart Lectures}

Under the will of the late $\mathrm{Mr}$. Herbert Akroyd Stuart a sum of $£ 700$ is held in trust by the Society for the offer of a prize every two years, for the best paper or lecture read or given before the Society dealing with the Origin and Development of Heavy-oil AeroEngines. The prize is open to members and non-members.

\section{Edward Busk Memorial Prize}

The Edward Busk Memorial Prize is offered annually for the best paper received by the Society on some subject of a technical nature in connection with aeroplanes (including seaplanes). Its value is twenty guineas.

\section{Pilcher Memorial Prize}

The Pilcher Memorial Prize is offered annually, at the discretion of the Council, for the best paper by a Student on heavier- than-air craft or any analogous subject. Its value is five guineas.

\section{Usborne Prize}

The Usborne Prize is offered annually, at the discretion of the Council, for the best paper by a Student on some subject in connection with Aero Engines. Its value is five pounds.

\section{Major Baden-Powell Memorial Prize}

The Major Baden-Powell Memorial Prize is awarded in May and December of each year to the Student who is considered the best student by the examiners in the Society's Association Fellowship examinations. Its value is three guineas.

\section{Elliott Memorial Prize}

The Elliott Memorial Prize is awarded twice yearly to the apprentice at Halton who has the highest percentage of marks in the passing-out examination.- Its value is two and a half guineas for each award.

\section{R. P. Alston Memorial Prize}

The R. P. Alston Memorial Prize is awarded to any Graduate or Student of the Society for work done leading to improvement in the safety of aircraft, and particularly for improvement in stability and control. Its value is approximately five pounds

\section{Branch Prize}

The Council offer an annual prize of twenty guineas for the best paper read before the Branches during the previous lecture Session. The prize is open to any member of the Society or of any Branch.

\section{Journal Premium Awards}

The Council has set aside an annual sum of $£ 250$ to be given as premium awards to authors of papers published in the Journal. 


\section{AN APPRECIATION}

The Council of the Royal Aeronautical Society wish to thank those Companies who, by their generous co-operation, have done so much to help in the production of the Journal

ACCLES \& POLLOCK LIMITED

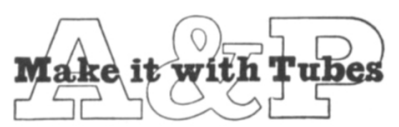

AEROPLANE \& MOTOR ALUMINIUM CASTINGS LTD.

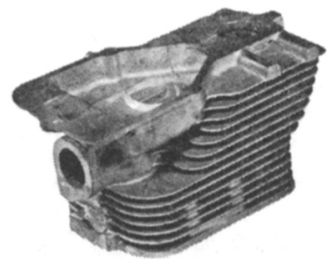

ALUMINIUM GRAVITY DIE CAST CYLINDER HEAD

AIRCRAFT MATERIALS LIMITED

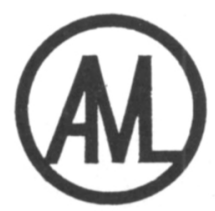

STRUCTURAL MATERIALS and COMPONENTS
AIRWORK LIMITED

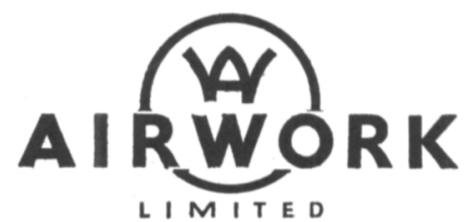

ALVIS LIMITED

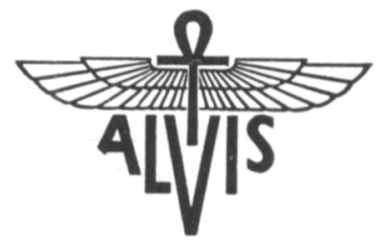

ARMSTRONG SIDDELEY MOTORS LTD.

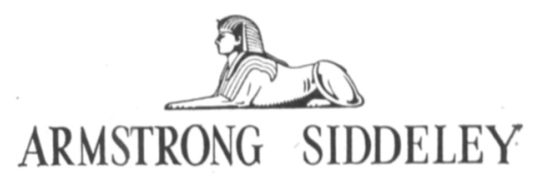

SIR W. G. ARMSTRONG WHITWORTH AIRCRAFT LTD.

SIR W. G. ARMSTRONG WHITWORTH AIRCRAFT LIMITED

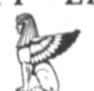




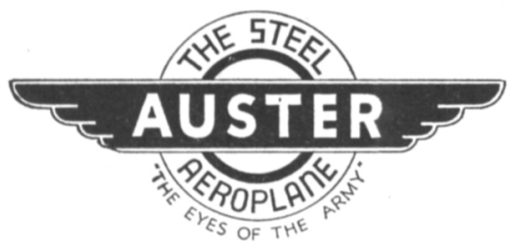

AUTOMOTIVE PRODUCTS COMPANY LTD.

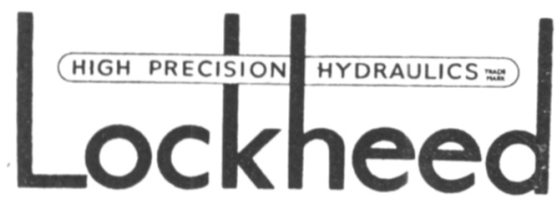

AVIMO LIMITED

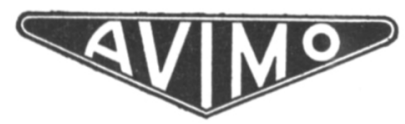

OPTICAL - MECHANICAL - ELECTRICAL INSTRUMENTS AERONAUTICAL EQUIPMENT

BAKELITE LIMITED

BAKELITE (8) PLASTICS

BIRMINGHAM ALUMINIUM CASTING (1903) CO. LTD.

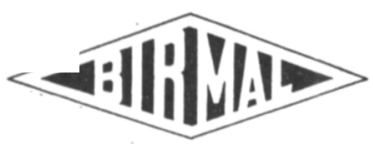

T. M. BIRKETT \& SONS LTD.

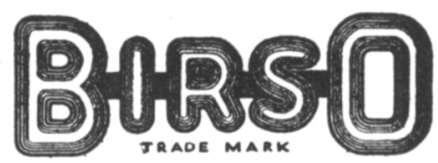

NON-FERROUS CASTINGS

AND MACHINED PARTS

HANLEY

STAFFS

BLACKBURN AIRCRAFT LTD.

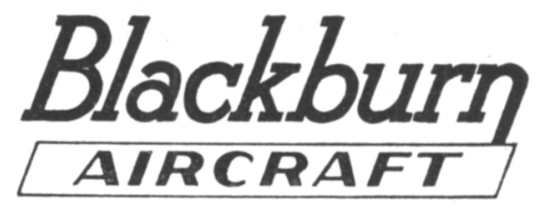

JAMES BOOTH G COMPANY LTD.

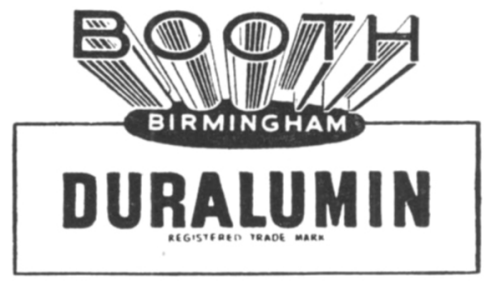

BOULTON PAUL AIRCRAFT LTD.

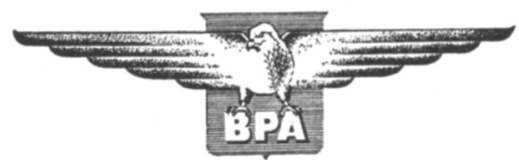

BRAKE LININCS LIMITED

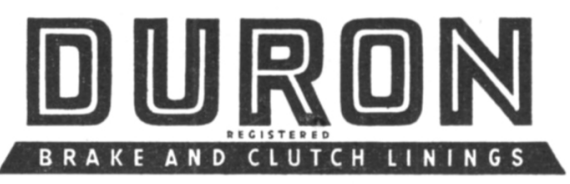


THE BRISTOL AEROPLANE CO., LTD.

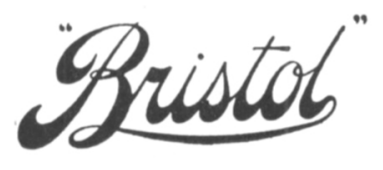

THE BRITISH ALUMINIUM CO., LTD.

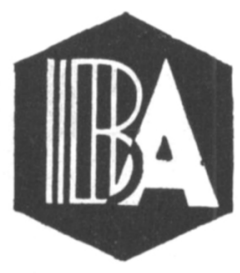

THE BRITISH AVIATION INSURANCE CO. LTD.

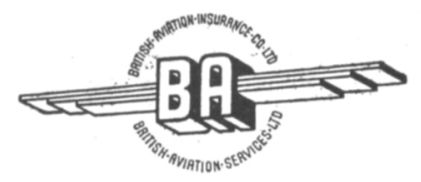

BRITISH AVIATION SERVICES LTD.

BRITISH INSULATED CALLENDER'S CABLES LTD.

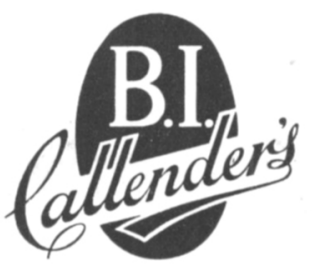

BROWN BROTHERS (AIRCRAFT) LTD.

\section{BrownBrothers AarrRafILtd.}

BRITISH OVERSEAS AIRWAYS CORPORATION

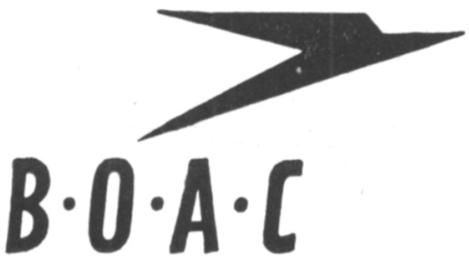

BRITISH THOMSON-HOUSTON CO. LIMITED (THE)

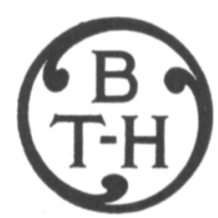

Magnetos and Electrical Equipment

BRITISH WIRE PRODUCTS LTD.

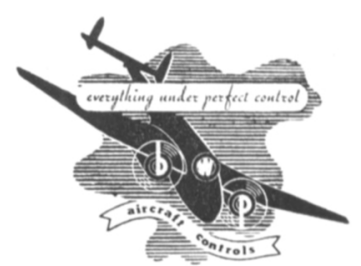

BROOM \& WADE LTD.

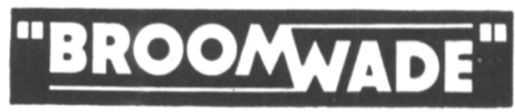

AND PNEUMATIC TOOLS
AIR COMPRESSORS

BUTLERS LIMITED

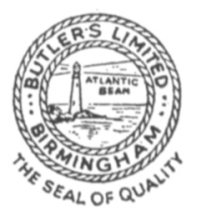

AUTOMOBILE, AIRCRAFT AND MARITIME LAMPS SEARCHLICHTS AND MOTOR ACCESSORIES

xxi 
THE CHLORIDE ELECTRICAL STORAGE CO. LTD.

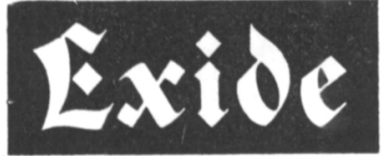

IAIRCRAFT BATTERIES

THE DE HAVILLAND AIRCRAFT COMPANY LTD.

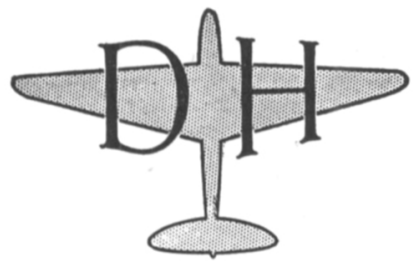

OELCO-REMY \& HYATT LTD.

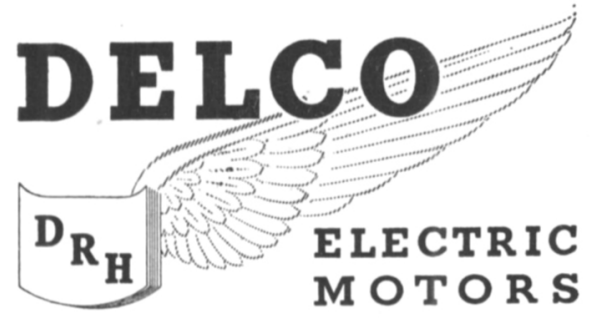

DOWTY EQUIPMENT LIMITED

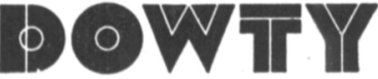

UNDERCARRIAGE AND HYDRAULIC EQUIFMENT

DUNFORD \& ELLIOTT (SHEFFIELD) LTD.

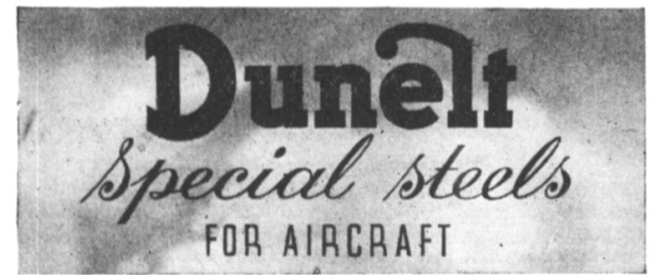

DUNLOP RUBBER CO., LTD.

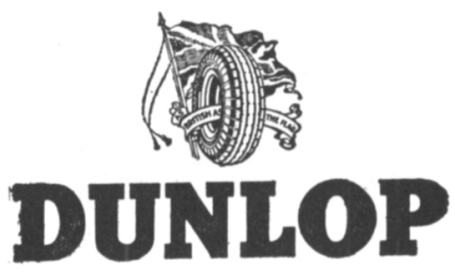

ENGLISH STEEL CORPORATION LTD.

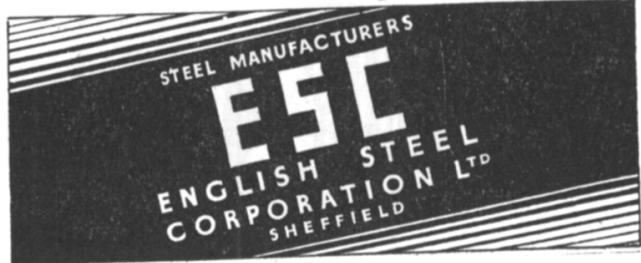

THE FAIREY AVIATION COMPANY LTD.

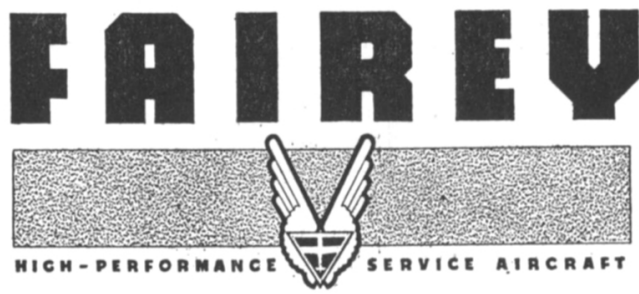

FIRTH-VICKERS STAINLESS STEELS LTD.

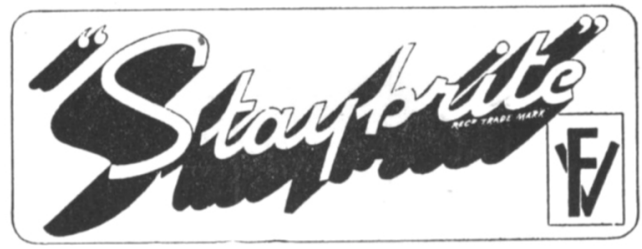

FOLLAND AIRCRAFT LIMITED

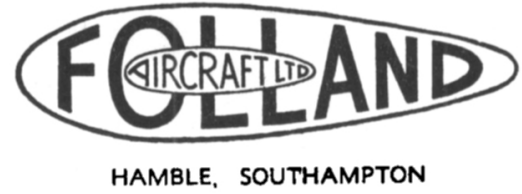




\section{$\Rightarrow$ \\ GFNERAL ARTRRPT ITD}

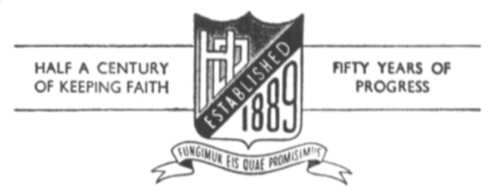

THE HESTON AIRCRAFT CO., LTD.

GLOSTER AIRCRAFT CO., LTD.
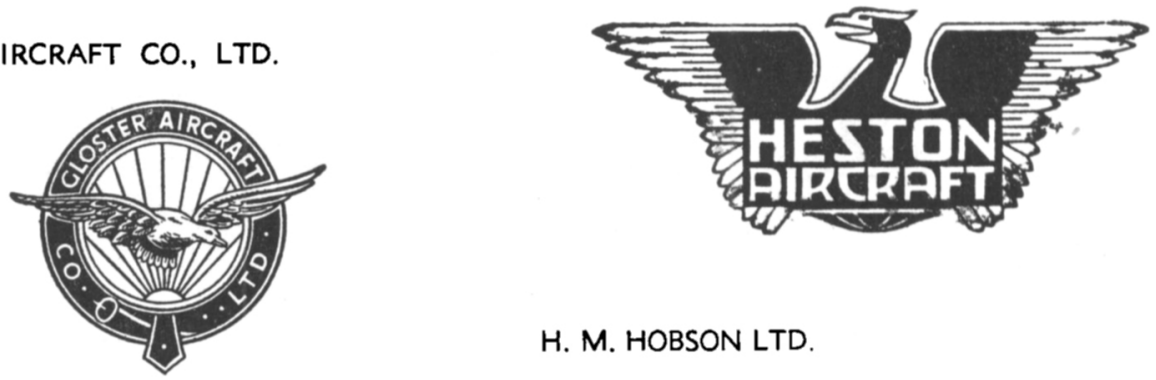

H. M. HOBSON LTD.

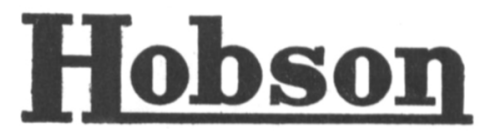

HANDLEY PAGE LIMITED

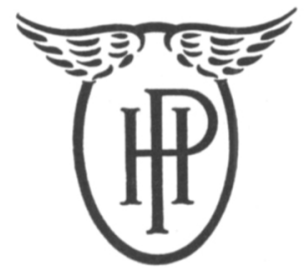

F. A. HUCHES \& CO., LIMITED

\section{ELEKTRON MAGNESIUM ALLOYS}
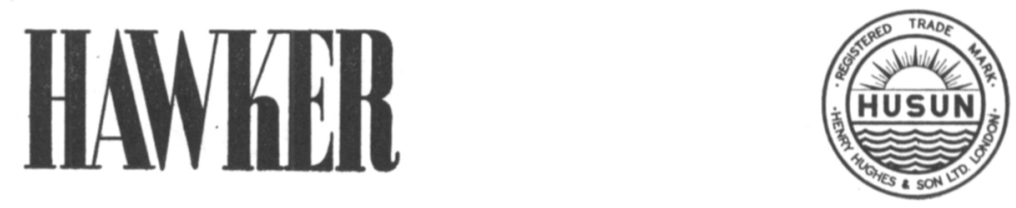

xxiii 


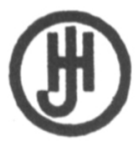

HUNTING AVIATION LIMITED

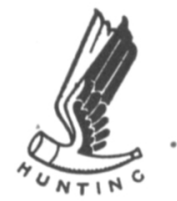

HYMATIC ENGINEERING CO. LTD.

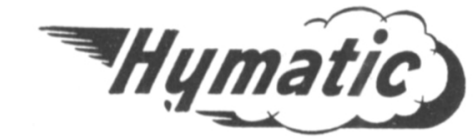

COMPRESSED AIR EQUIPMENT

IMPERIAL CHEMICAL INDUSTRIES LTD.

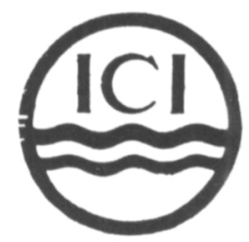

INTEGRAL LIMITED

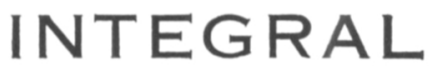

HYDRAULIC PUMPS

A N D EQUIPMEN T

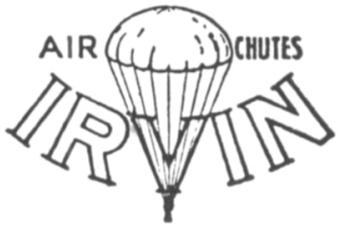

K.L.G. SPARKING PLUCS LTD.

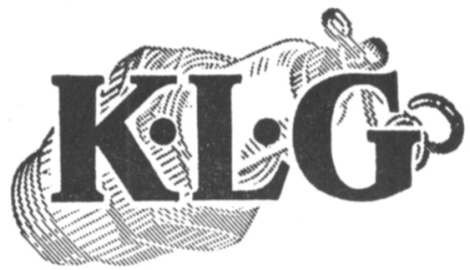

KELVIN BOTTOMLEY \& BAIRD LTD.

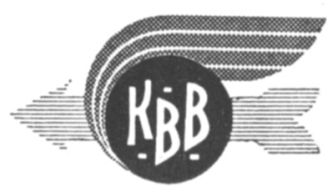

LIGHT-METAL FORGINCS LTD.

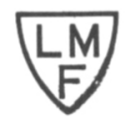

LODCE PLUGS LIMITED

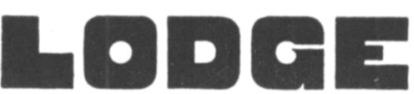

P L U G S

xxiv. 


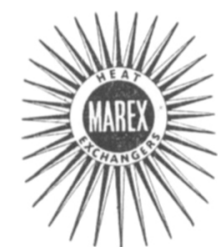

MILES AIRCRAFT LIMITED

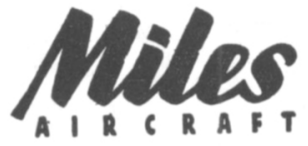

MOLLART ENGINEERING COMPANY LTD.

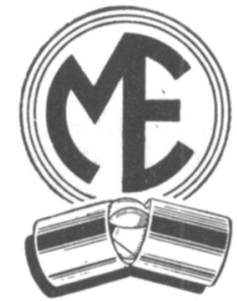

Members of the IGauge and Tool Makers' Association

THE MOND NICKEL COMPANY LTD

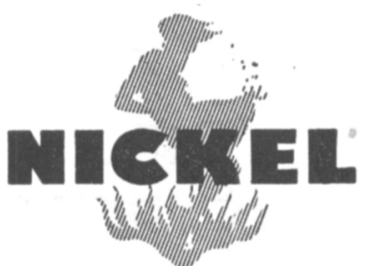

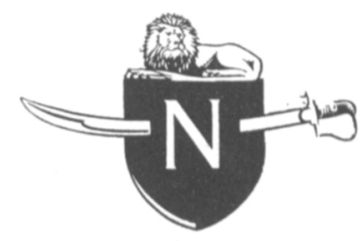

NORMALAIR LIMITED

NORMALAIR

THE PALMER TYRE LIMITED

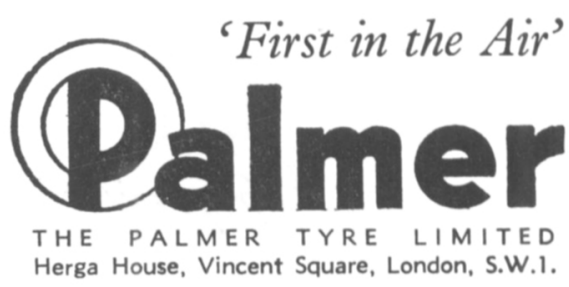

PERCIVAL AIRCRAFT LIMITED

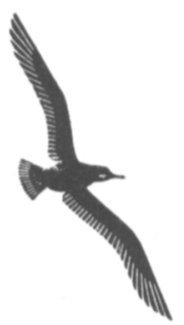


PETO \& RADFORD

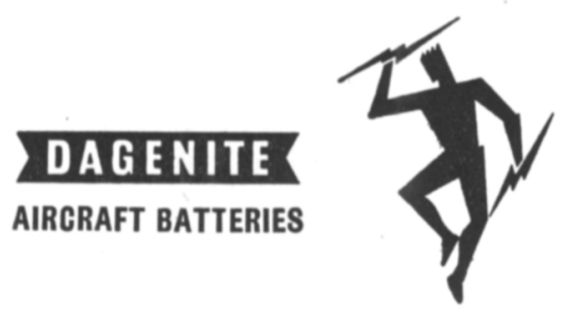

PORTSMOUTH AVIATION LIMITED

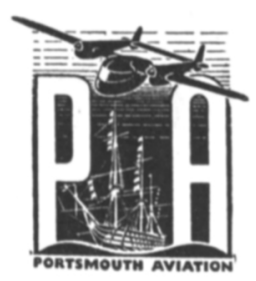

PRESSED STEEL CO., LIMITED

\section{PRESTCOLD \\ Aefigeration}

QANTAS EMPIRE AIRWAYS

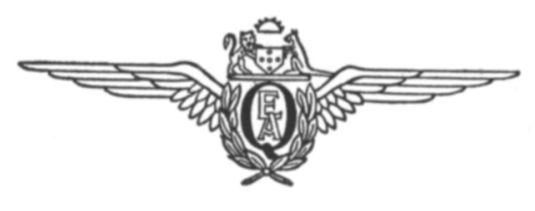

R.F.D. COMPANY LTD.

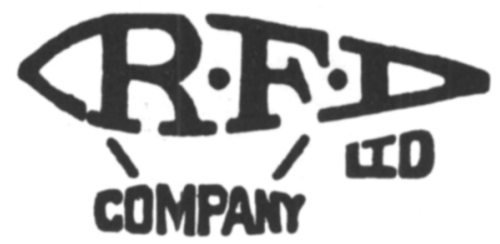

A. V. ROE \& COMPANY LIMITED

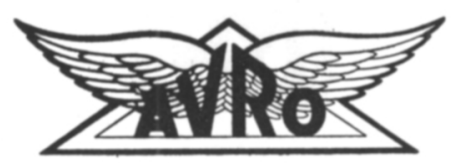

THE PULSOMETER ENCINEERINC CO. LTD.

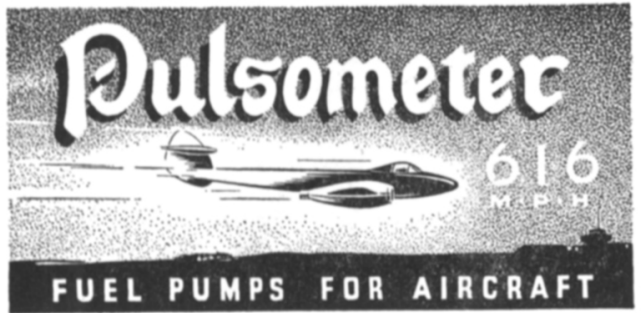

ROLLS-ROYCE LIMITED

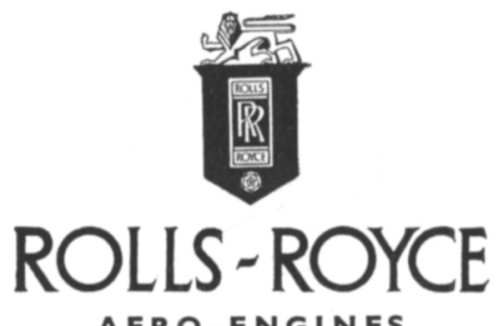

AERO-ENGINES 
ROTAX LIMITED

\section{IDTAX}

AIRCRAFT ELECTRICAL ENGINEERS

ROTOL LIMITED

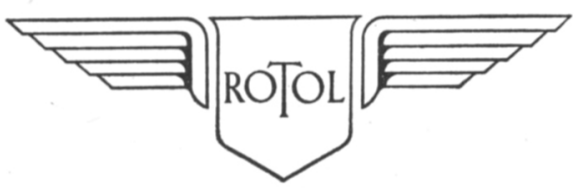

SANGAMO WESTON LTD.

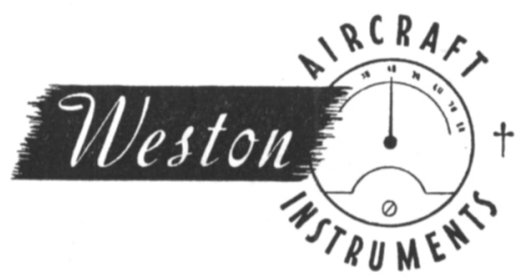

SERCK RADIATORS LIMITED

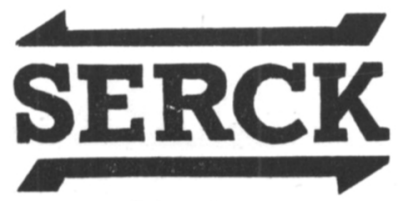

SHORT BROTHERS (ROCHESTER \& BEDFORD) LIMITED

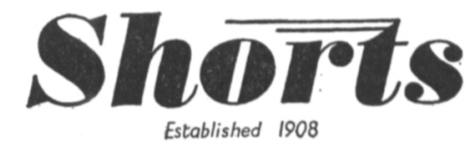

THE FIRST MANUFACTURERS

OF AIRCRAFT IN THE WORLD

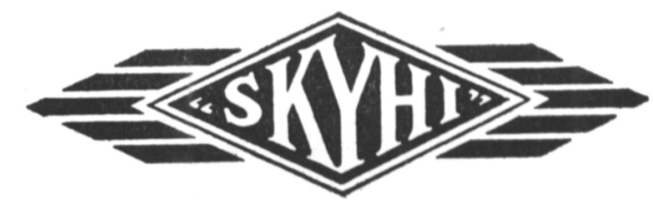

SMITHS AIRCRAFT INSTRUMENTS LTD.

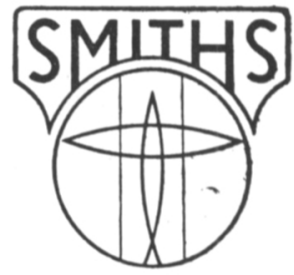

THE SPERRY GYROSCOPE COMPANY LTD.

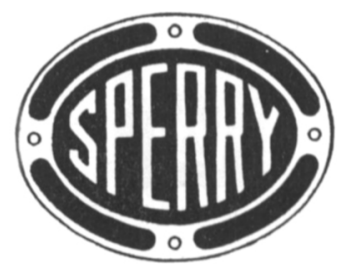


THE TECHNOLOGICAL INSTITUTE OF GREAT BRITAIN

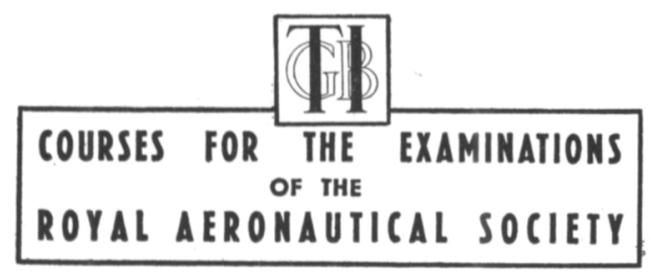

THE UNITED STEEL COMPANIES LIMITED

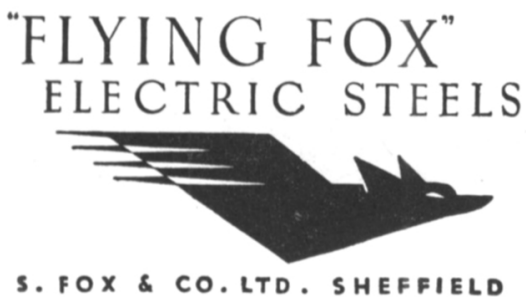

VOKES LIMITED

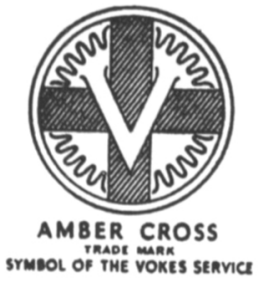

WESTLAND AIRCRAFT LIMITED

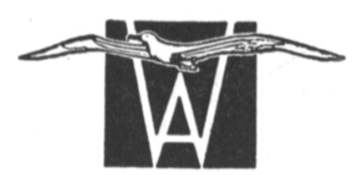

A. C. WICKMAN LIMITED

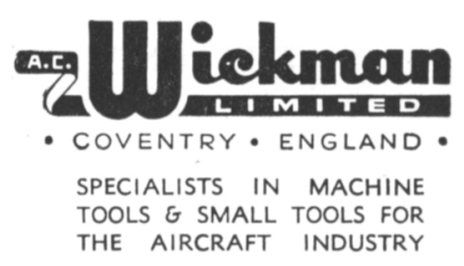

HENRY WICGIN \& COMPANY LTD.

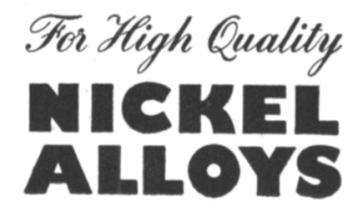

WILLIAMSON MANUFACTURING CO., LTD

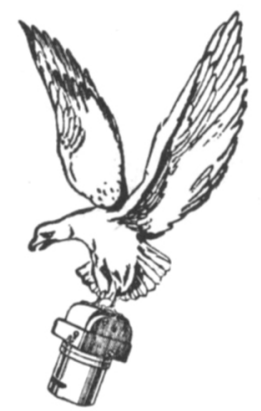

YORKSHIRE ENGINEERING SUPPLIES LTD.

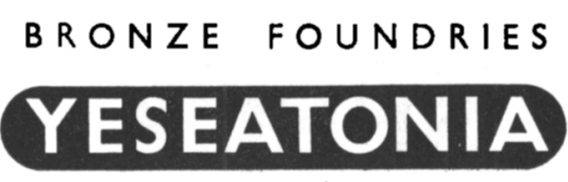

BEARINGS AND BUSHES 


\section{DIRECTORY OF ADVERTISERS}

ACCLE \& POLLOCK LTD.

ACCLES \& POLLOCR LTD. $\ldots$ M C LTD. $\ldots \quad \ldots \quad \ldots \quad \cdots \quad \cdots \quad \cdots$

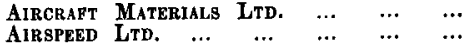
$\begin{array}{llllll}\text { AlRSPEED LTD. } & \ldots & \ldots & \ldots & \ldots & \ldots \\ \text { AIR WORK LTD. } & \ldots & \ldots & \ldots & \ldots & \ldots\end{array}$ ALBRIGHT \& WILSON $\cdots$

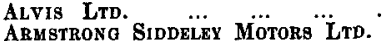
ARMSTRONG WHITWORTE AIRCRAFT, SIR $\ddot{W}$

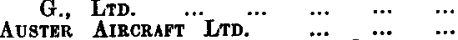
AUSTER AIRCRAFT LTD. $\begin{array}{llll}\text { EQUIPMENT Co. LTD. } & \ldots & \ldots & \ldots \\ \end{array}$ $\begin{array}{lllll}\text { Automotrve Prodvets Co. Ltd. } & \ldots & \ldots \\ \text { Avimo Ltd. }\end{array}$

BaEFLITR LTD.

BIRKETt, T. M. $\dddot{*}$ SoNs Lto $\ldots \ldots$ Co. LTD.

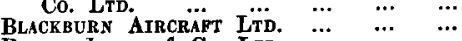
BOOTH, JaMES, \& Co. LTD. $\ldots . \quad \ldots$. Boulton Pavl alRcraft Lto. $\ldots$. Beart Livivas LTD. BRISTOL ABRoplane Co." Ltd.," The"

British AlUminidm Co. Lto.

British Aviation Insoraster Co. Z̈td. ... British A viation Services Ltd.

British Belting and asbestos L $\ldots$

British European Airways Corporation British Irsulated Callender's Cables LRD.

British Overseas airways Corporatiox

British Thomson-Houston Co. LTD. British Wire Products Lid.

BROOM \& WADE LTD.

BROOM \& WADE LTD. $\ldots$... $\ldots$ Butlers LTD.

Chloridg Electrical Storage Co. Lto.,

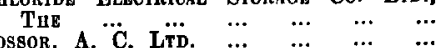

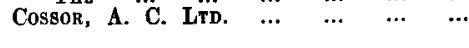

De Haviluand aircraft Co. LTD., Thz Decca NavigatoR Co. Ltd. ... $\ldots . .7$

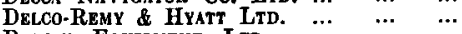
DOWTY EQUIPMENT LTD.

DUNFORD \& ELEIOTT (SHEFFImL) LTD. $\ldots$

DUNLOP RUBBr Co.

Englisi Sterl Corporation Lto.

Fairey Aviation Co. Lto.

Firth, Thos., \& John Brown Li... Firte-Vickers Stainless Steels Ltd. ... Follind AIRCBART LTD.

General Aircraft LTd. Gloster Aircratt Co. Ltd.

Handley Page Lto HAWRER AIRCRAFT LTD. Hawker SiddrLey

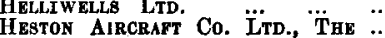

foBson, H. M. LTD.

Hognes, F. A \&. Co. ITo.

Hughes, HeNry, \& Son I

Hughes-Johnson Stampings Ltd., The ..

Hunting Aviation Lto. $\ldots$... $\ldots$

Hymatic Evgiverering Co. LTo.
Paddock Works, Oldbury, Birmingham

Wood Lane, Erdington, Birmingham

Midland Road, London, N.W.1. $\quad \ldots .6$.

The Airport, Portsmouth $\ldots \begin{array}{lllll}\ldots & \ldots & \ldots & \ldots & \ldots\end{array}$

15 Chesterfield Street, W.1. 49 Park Lane, W.1. Works : oldbury and Widnes.

Holyhead Road, Coventry $\ldots$... $\ldots$....

Parkside, Coventry

Whitley, Coventry $\ldots \ldots$ Whitannia Worke, Thurmaston, Ieicester

Winder House, Douglas Street, S.W.1 ...

Tachbrook Road, Leamington Spa $\ldots$...

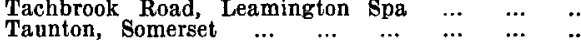

18 Grosvenor Gardens, S.W.1

Thomas

Birmid Works, Smethwick, Birmingham $40 \quad \ldots \quad$... Smethwick 1431

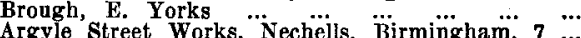

$\begin{array}{llllll}\text { Wolverhampton, Staffs } \quad \ldots & \ldots & \ldots & \ldots & \ldots\end{array}$

Buxton, Derbyshire

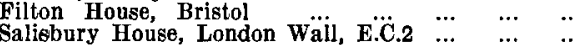

3-4 Lime Street, London Wall, E.C.2 $\ldots$

Lim Street, E.C.3

Cleckheaton, Yorks.

59 Southwark Street, "

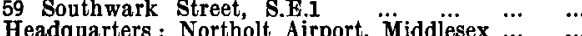

Main Works : Erith, Helsby, Leigh, Lancs. and

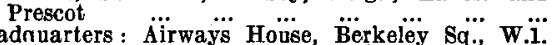

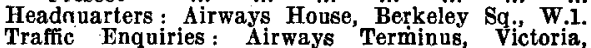

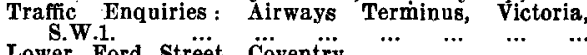
Lower Ford Street, $\begin{array}{cccccc}\dddot{\text { Coventry }} & \cdots & \cdots & \ldots & \ldots\end{array}$

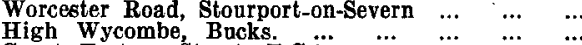

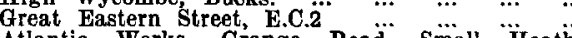
Atlantic Works, Grange Road, small \#eath,

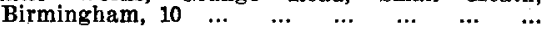

Exide Works, Clifton Junction, near Manchester ... Highbury Grove, N.5 $\quad \ldots \quad$...

Swinton 2011

Canonbury 1234

Hatfield Aerodrome, Herts.

I-3 Brixton Road, S.W.9.

111 Grosvenor Road, S. W

Attercliffe Wharf Works, sheffield, $, \cdots, \quad \cdots \quad$,

Fort Dunlop, Erdington, Birmingham, $24 \quad \ldots$.

Vickers Works, Sheffield

Brough 121 1221

Fordhouses 3191

Buxton 776

Clerkenwell 3494

Mansion House 0444

Paddington 7040

Cleckheaton 800

Hop 0192

Ruislip 6061

Mayfair 8400

Victoria 2323

Coventry 4104

Stourport 240

High Wycombe 1630

Bishopsgate 7654

Victoria 2164-6

Hatfield 2345

Reliance 3311

Victoria 6242

Cheltenham 53471

Sheffield $41121-4$

Erdington 2121

Sheffield 41071

Hayes, Middlesex $\ldots$.. $\begin{array}{llllll} & \ldots & \ldots & \ldots & \ldots & \text { Hayes } 3800\end{array}$

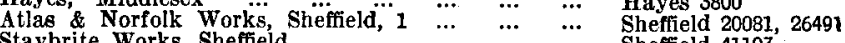

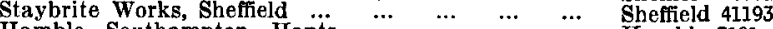

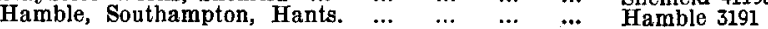

The London Air Park, Feltham, Middlesex ... Feltham 3636 Gloster Works and Aerodrome, Hucclecote, Glos.

Gloucester 6294

Cricklewood, N.W.2

Canbury Park Road, $\dddot{K}$ ingston-on-Thames $\quad \ldots \quad$...

Gladstone 8000

Kingston 1044

Abbey 2672

Stratford-on-Avon 3265

Southall 2321

Fordhouses 2266

Welbeck 2332-6

Hainault 2601

Hainault 2601

Broadwell 1361

Redditch 743 


\section{IRECT OR Y OF A DVERTISERS}

\begin{abstract}
ILIFFE AND SoNs LTD. ...
IMPERIAL CIEMICAL INDUSTRIES LTD. ...

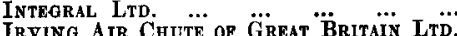

K. I G Sareing Putgs LTD.

Kelvin, BotTonley \& Baird Ltd. $\ldots$.

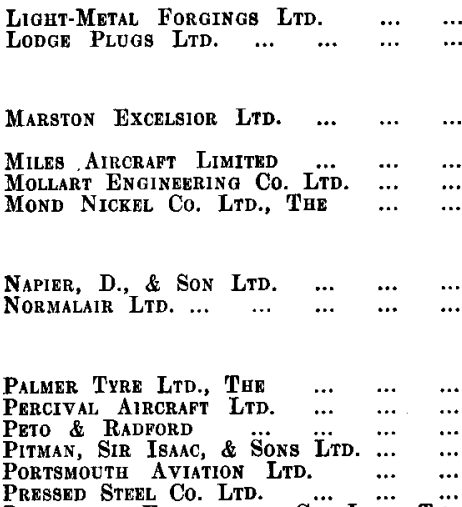

Pressed Stegl Co. Ltd.
Pulsometer Enginering
Co. Lto., Tä
\end{abstract}

Dorset House, Stamford Street, S.E.1

London, S.W.1 $\ldots$... $\ldots$.

Ickneild Way, Letchworth, Herts.
Waterloo 3333 Victoria 4444

Wolverhampton 24984-6

Letchworth 888

utney Vale, S.W.15

Kelvin Alenu

$\begin{array}{llllll}\text { Kelvin } & \text { Avenue, } \\ \text { Kelvin Whilington, Glasgow, } & \text { S.W.2 } & \ldots & \text { Half way } 3331 \\ \text { Works, Basingstoke } & \ldots & \ldots & \ldots & \ldots & \text { Basingstoke } 690\end{array}$

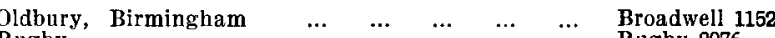

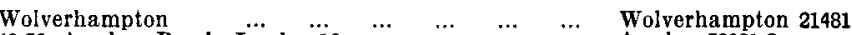

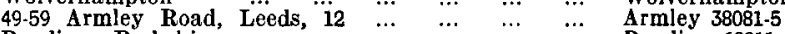

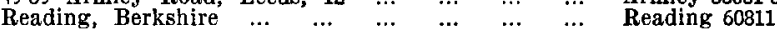

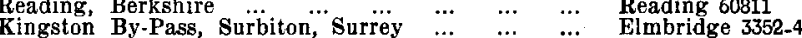

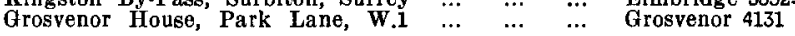

\section{QANTAS EmplRe AIRWays \\ Terminal, Buckingham Palace Road, S.W.1 ... Victoria 3126

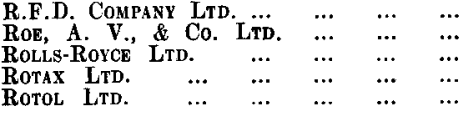 \\ Sangamo Weston LtD.

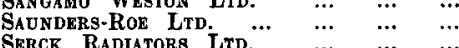 Short Brothers (Rochigter $\cdots$ \& Bëporö) \\ LTD. $\quad \ldots \quad \ldots \quad \ldots \quad \ldots \quad \ldots \quad \ldots$

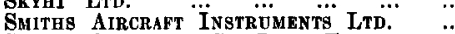 \\ SPerry Gyroscope Co. LtD., THE ... ... \\ Stoke Road, Guildford, Surre \\ Newton Heath, Manchester \\ Derby \\ Willesden Junction, N.W.10 \\ Cheltenham Road, Gloucester \\ Guildford 3232 \\ Failsworth 2020 \\ Derby 2424 \\ Elgar 7777 \\ Gloucester 4431 \\ Great Cambridge Road, Enfield, Midd'esex ... 49 Parliament Street, Westminster, S.W.1 ... Warwick, Road, Birmingham, ll $\ldots$... \\ Rochester, Kent \\ Great West Road, Brentford, London $\ldots$... "Skyhi" Works, Worton Rd., Isleworth, Mïddesex Cricklewood Works, London, N.W.3 \\ Great West Road, Brentford, Middlesex \\ Enfield 3434 \& 1242 \\ Whitehall 727 \\ Victoria 0531 \\ Chatham 2261 \\ Ealing 2212 \\ Hounslow 2211 \\ Gladstone 3333 \\ Ealing 6771}

Acton, W.3

West Hendford, "' Yeovil, Somerse

Herga House, Vincent Square, \$.W.1

Luton Airport, Luton, Beds.

Chequers Lane, Dagenham, Easex

Parker Street, Kingsway, W.C.2

The Airport, Portsmouth

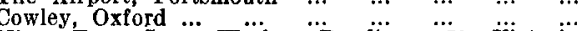

Nine Ems Iron Works, “Reading; ${ }^{3} 9$ Victoria

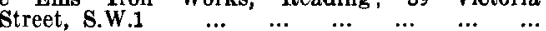

Technological Institute of Great

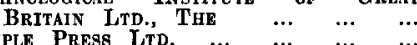

United Steel Companies Ltd., The

Vickers-ARMSTRONGg Lrd.

VOKES LTD.

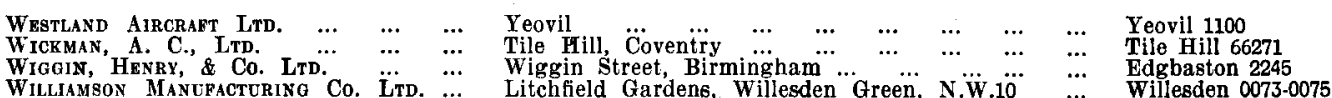

Yorkshire Engineering Supplies LtD. Bronze Foundries, Upper Wortley Road, Leeds, 12 Leeds 38234 \& 38291 


\section{JOURNAL PREMIUM AWARDS}

The Council have set aside an annual sum of $£ 250$ for the award of premiums for papers published in the Journal and the Council hope that members (or non-members) will contribute papers on their own special subjects.

\section{CONTENTS OF THE AUGUST JOURNAL}

The Development of the Goblin Engine, by E. S. Moult,, B.Sc., M.I.Mech.E., A.F.R.Ae.S.

Useful Load Ratio with Jet and Airscrew Propulsion of Aircraft, by Bo K. O. Lundberg, A.F.R.Ae.S.

Stability of an Aircraft Structure in a Strength Test Frame, by P. B. Walker, M.A., Ph.D., F.R.Ae.S.

\section{ANGLO-AMERICAN AERONAUTICAL CONFERENCE}

Members are reminded that application forms for membership of the Anglo-American Aeronautical Conference, 3rd-6th September, were enclosed with the JUNE Journal and Monthly Notices. Eighteen papers will be read and discussed during the Conference. These papers will not be published in the Journal, but will be published separately.

\section{GARDEN PARTY AND GUILDHALL DINNER}

Members are reminded that application forms for tickets for the Garden Party (Radlett, 14th September, 1947) and for the Dinner at Guildhall (9th September, 1947) were enclosed with the JULY Journal and Monthly Notices. Early, application is advised for both functions as the numbers to either will be strictly limited and cannot be exceeded in any circumstances.

\section{ELLIOTT MEMORIAL PRIZE}

The Elliott Memorial Prize has been awarded to Corporal Air Apprentice A. E. Dyer for the highest marks obtained by the August 1944 Entry in the General Studies Examination.

\section{CLOSING OF THE LIBRARY DURING AUGUST}

The Library will be closed for stocktaking from 11th to 23rd August inclusive. Members are requested to return all books which they have on loan from the Library not later than Saturday, 9th August.

\section{MEETINGS HELD IN THE OFFICES OF THE SOCIETY}

Meetings have been held during the month in the offices of the Society by the Aeronautical Research Council, Aerodrome Owners' Association, Society of British Aircraft Constructors, Meteorological Research Committee, R.A.E. J.A.C. Materials Committee, Air League of the British Empire, Graduates' and Students' Section. 


\section{GLOUCESTER AND CHELTENHAM BRANCH}

A visit has been arranged to the Bristol Aeroplane Co. Ltd. to inspect the Brabazon I on Saturday, 23rd August, at 2.30 p.m. The party is limited to 100. Applications should be made to the Hon. Secretary, J. F. Cuss, A.M.I.Mech.E., A.F.R.Ae.S., c/o Gloster Aircraft Co. Ltd., Witcombe, Glos., or to a Committee member.

\section{READING BRANCH-GARDEN PARTY}

The Reading Branch Garden Party will be held on Saturday, 6th September, 1947, from 2.30 p.m. until about 6 p.m. There will be a Grand Fly Past at about 5 p.m.

A limited number of tickets will be available at $5 /-$ per head to all members of the Society and to the Branches of the Society. The tickets will include tea and car park and/or Aircraft park. There will be no landing fees for aircraft which arrive before 2 o'clock. Service will be available for such aircraft.

Applications for tickets should be made, with remittance, to the Secretary, Reading Branch:-B. Buck, Esq., A.F.R.Ae.S., Miles Aircraft Ltd., The Aerodrome, Reading, Berks.

\section{GRADUATES' AND STUDENTS' SECTION}

\section{Discussion}

A discussion on the "Structural Design of Wings" will be held at 7.30 p.m. on Tuesday, 23rd September at the Society, 4 Hamilton Place, W.1. The Discussion will be introduced by Mr. F. Tyson, A.F.R.Ae.S., Assistant Chief Stressman; Handley Page Ltd.

\section{Visits}

30th August, 1947 (Saturday)_A visit has been arranged to the works of Miles Aircraft Ltd., Reading, at 2.30 p.m.

17 th September, 1947 (Wednesday) - A visit has been arranged to the works of the de Havilland Aircraft Co. Ltd. at 2.30 p.m.

\section{Dance}

The committee have pleasure in announcing that the Section's Annual Dance, open to members and their friends, will be held on Friday, 17th October at the Royal Hotel, Woburn Place, W.C.1. Further details will be published in the September issue of the Journal.

\section{CHANGES OF ADDRESS}

To assist in keeping the records of members correct and up to date the Secretary will be glad if all members will notify him as soon as possible of changes of address.

When notifying changes please give the following particulars:-

Name (in block letters).

Grade of membership.

New address (in block letters).

Old address.

Changes of address must be received before the 15th of the month in order to be eifective for the Journal for the following month. 


\section{ASSOCIATE FELLOWSHIP EXAMINATION RESULTS}

The following were the successful candidates in the Associate Fellowship Examination held in May, 1947:-

J. M. Allwright, Design (Aero-Engines); G. E. Archdale, Strength of Aeronautical Materials and Structures (First Place), Design (Aircraft); P. W. Baker, Applied Mathematics, Theory of Internal Combustion Engines, Design (Aero-Engines) (First Place); D. F. Barnes, Applied Mathematics, Strength of Aeronautical Materials and Structures; P. B. Benham, Applied Mathematics; A. Berrington, Pure Mathematics; G. B. Bhide, Aerodynamics; G. Boyce, Theory of Machines; P. A. Budden, Aerodynamics; J. J. Bukovsky, Air Transport; "H. Busen-Schmidt, Theory of Internal Combustion Engines (First Place); S. A. Caplan, Pure Mathematics; H. K. Cartwright, Pure Mathematics; L. J. Chantler, Pure Mathematics, Theory of Machines; W. G. Clennett, Pure Mathematics (First Place), Strength of Aeronautical Materials and Structures; Aircraft Materials; J. R. Combley, Aerodynamics, Design (Aircraft); H. Cook, Design (Aero-Engines); T. N. Corkhill, Applied Mathematics, Strength of Aeronautical Materials and Structures, Aerodynamics; E. Coton, Applied Mathematics, Aerodynamics, Meteorology; B. G. Cour-Palais, Theory of Internal Combustion Engines; E. N. Crabbe, Aerodynamics; K. D. Crisp, Pure Mathematics, Theory of Machines (First Place); N. S. Currey, Strength of Aeronautical Materials and Structures; Miss J. L. Davis, Pure Mathematics, Strength of Aeronautical Materials and Structures; E. D. Dixon, Pure Mathematics; J. Dolezal, Aerodynamics (First Place), Air Transport (First Place); J. Dorsey, Aircraft Materials; A. A. Down, Applied Mathematics; F. A. Drayton, Aircraft Materials; Miss J. R. Duncan, Pure Mathematics; G. D. Evans, Theory of Machines; A. Fairbrother, Aerodynamics; P. L. E. Gallimore, Aerodynamics; F. R. Gibb, Applied Mathematics, Strength of Aeronautical Materials and Structures, Aerodynamics; Miss D. Gwynne, Pure Mathematics; N. T. W. Harper, Theory of Internal Combustion Engines; L. H. W. Harris, Applied Mathematics; P. A. Hatswell, Theory of Internal Combustion Engines; J. M. Johnston, Applied Mathematics, Strength of Aeronautical Materials and Structures, Design (Aircraft); J. G. Jones, Applied Mathematics (First Place); J. B. E. Keeble, Pure Mathematics; E. D. King, Theory of Machines; R. E. Lambert, Theory of Machines; R. A. Langley, Pure Mathematics, Aerodynamics, Theory of Internal Combustion Engines; M. P. Le Lohe, Applied Mathematics; A. Lewandowski, Design (Aero-Engines); K. Macewicz, Design (Aero-Engines), Theory of Machines; S. H. Masters, Aircraft Materials; R. L. McCallum, Design (Aero-Engines); M. P. McHeffey, Applied Mathematics, Strength of Aeronautical Materials and Structures, Design (Aircraft); G. McIntosh, Strength of Aeronautical Materials and Structures, Aerodynamics; A. Measures, Strength of Aeronautical Materials and Structures; J. A. S. Moir, Pure Mathematics; E. Mucha, Theory of Internal Combustion Engines, Design (Aeró-Engines); U. N. Nayak, Theory of Internal Combustion Engines; G. F. Perry, Strength of Aeronautical Materials and Structures; J. W. Piggott, Meteorology and its Application to Aeronautics; K. G. Rendle, Strength of Aeronautical Materials and Structures; G. C. Ricketts, Aerodynamics; J. H. Risdon, Theory of Machines; G. I. Robinson, Applied Mathematics, Strength of Aeronautical Materials and Structures, Design (Aircraft); R. R. Robinson, Pure Mathematics, Strength of Aeronautical Materials and Structures, Aircraft Materials; R. C. Rogers, Theory of Internal Combustion Engines, Aircraft Materials, Applied Mathematics; J. G. Romeril, Theory of Internal Combustion Engines, Design (Aero-Engines); D. N. Scard, Applied Mathematics, Aerodynamics; L. J. A. Sice, Applied Mathematics; L. Stern, Theory of Internal Combustion Engines, Theory of Machines; C. G. A. Stillaman, Aircraft Materials; 
R. Szymanski, Applied Mathematics, Design (Aero-Engines); D. L. Taylor, Pure Mathematics; J. B. Thompson, Pure Mathematics; Titmuss, H., Strength of Aeronautical Materials and Structures; J. A. Tupman, Applied Mathematics, Design (Aircraft); R. A. Vair, Pure Mathematics, Strength of Aeronautical Materials and Structures, Aircraft Materials; J. H. WaImsley, Design (Aircraft); J. Walmsley, Pure Mathematics.

\section{ELECTION OF MEMBERS}

\section{Fellows}

Henry Paterson Fraser (from Associate Fellow), Stanley George Hooker (from Associate Fellow).

\section{Associate Fellows}

Rowland William Angell, Leslie George Bednell, William Norman Blacklock, Frederick Wiliam Robert Bird (from Associate), Francis Charles Bradley (from Associate), Geoffrey Frank Briginshaw (from Graduate), Richard Henry Corthorn Brousson (from Graduate), Cyril Douglas Brown, Cecil John Carter, Horace William Clarke, Athol Henry Fear, Albert Henry Figg (from Graduate), Arthur Ernest Fishlock (from Associate), Frank Leslie Garton, Maurice Alfred Glasspole (from Associate), Leonard Edgar Good, Douglas Gerald Seafield Grant (from Associate), Frederick Rowland Grey, Frederick John Hamilton, Saleh Helmy; Victor Alexander Higgs, Martin Owen Hook, Eric Stanley How (from Graduate), Stanley Peerman Hutton, Thomas Albert Knight, Alfred Stanley Knowles, Mark Dawson Knowles, Peter Stevens MacGregor, Bernard Walter Martin (from Graduate), Harry Maynard (from Graduate), Mahadeo Vinayak Namjoshi, Christopher Bernard Vere Neilson, Sidney Joseph Paine (from Associate), John Joseph Parkes, Cyril Henry Penn, Courtland Davis Perkins, Frederick Noel Pierce, Gordon Powell, Arthur Alfred Puttick, Norman Ring (from Student), Philip Lawton Sumner, Ronald Tatham, Roy Albert Tier (from Student), Frank Lionel Tollow, Cyril Frederick Turner (from Associate), Raymond Hedley Vernon (from Graduate), Reginald Thomas Weatherstone (from Graduate), Francis James Prince Whiting.

\section{Associates}

Frederick George Banting, Robert William Warren Buckley, Cyril George Butler, Frank Dixon, Denis Edward Hastwell, Alec Henry Jones, John Gordon Lacey, James Bertram Linton, George Alfred Bertram Lord, Patrick J. McEnery, Ralph Wardle Moore, Michael Pierre Perrelet, Theodore Grimmer Raynham (from Student), Frederick Victor Rehill (from Student), William Robb, Gerard Claughton Roberts, Eric William Saben (from Student), Albert Ernest Trevelyan, Denis Frederick Woor, Geoffrey David Puget Worthington.

\section{Graduates}

Derek George Ashwell, William Andrew Baker, Harold Charles Beasley, Alan Edmund Clarke, Douglas Ronald James Colpus (from Student), James Arnot Hamilton, Alfred Dennis Jackson (from Student), Aubrey Frederick Walter Langford, John Rutter Lloyd, Lawrence Halford Warren, Ellis Luckraft 'from Student), Donald Pratt, John Anthony Staunton, Peter John Vinson (from Student).

\section{Students}

Charles Crawford Aitkenhead, Denzil Arthur Ashover, Arthur Francis Becker, Frank Blamire, John Lawrence Budd, Roland Frederick Burgers, Anthony Lambert Cole, Eric Lewis Eavis, William M. Giffen, Derek Michael Gladstone, Harold Frederick Hawkins, 
Reginald Arthur Hinkley, William Donald Horsfield, Denis Lawrence Lofts, David Muir Mackie, James Parker, David Windsor Parry, Julien Podolski, Peter Robinson, Christophır John Rogers, Ronald Leon Samuels, Kenneth Robin Warren.

\section{Companion}

John Michael Storer Keen.

\section{ACKNOWLEDGMENT}

The Council acknowledge with grateful thanks the gift of Aeronautica by Monck Mason and a collection of lantern slides from G. W. Simmons, Esq.

\section{ADDITIONS TO THE LIBRARY}

Pamphlets in italics with location refèrence following in brackets. Books marked * or ** may not be taken out on loan.

A.a.333-Theory of Flight. Richard von Mises. McGraw Hill. 1945.

B.b.73-Principles of the Helicopter. Dr. Alexander Klemin. Reprint from Aero Digest. Y.3.ivI.

BB.c.105-Aircraft Propeller Design. F. T. Meacock. Spon. 1947.

E.d.31_British Standards for Workshop Practice. B. S. Handbook No. 2. British Standards Institute. 1946.

G.f.33-The story of electric welding. Published by "Sheet Metal Industries" for British Electric \& Allied Manufacturers' Association. 1947.

G.e.E.65-Symposium on the Hardenability of Steel. The Iron \& Steel Institute. 1946.

G.e.E.66-Crucible Cast Steel. John Vessey \& Sons. Sheffield. 1947.

H.b.92.-The adequacy of the passenger liability limits of the Warsave convention of 1929. J. Brooks, B. Parker. Reprint from Journal of Air Law \& Commerce. Vol. 14. No. 1. 1947. (P.H.1.).

L.d.116-Notices to Airmen. Nos. 156, 157, 158, 159, 160, 161, 162, 165, 166, 167, 168, $169,170,171,172,173,174,175,176,177,178,179,180,181,182,183,184,185$, $186,187,189$.

*Q.a.173 - Catalogue Générale du Centre de Documentation Aéronautique Internationale. Aero Club de France. 1944.

*Q.a.174_Library Catalogue. 1946. The Bristol Aeroplane Co. 1947.

UC.5/33 - C.S.I.R. Division of Aeronautics. Report S.M.82. The strength of machine parts under fluctuating loads. 1946. M.S. Paterson and H. A. Wills. Aeronautical Laboratory, Melbourne.

UC.5/34-C.S.I.R. Division of Aeronautics. Report S.M.86. On a method of increasing the rate of convergence of some iteration processes. J.P.O. Silberstein. Aeronautical Laboratory Melbourne. 1947.

*WB.17/18-Nickel Bulletin. Vol. 18, 1945 Mond Nickel Co. London.

\section{A.R.C. Reports and Memoranda-}

2104-A mathematical method of cascade design. M. J. Lighthill.

2097-A note on the interpretation of V.g. records. P. E. Montagnon, A. Robinson and S. V. Fagg.

2126-The "china clay" method of indicating transition. E. J. Richard and F. $H$. Burstall. 
2131_N. P. L. Mach number gauge. D. Giles.

2044-_Velocities and speeds through a shock wave. A. Thom and P. R. Owen.

2114-The representation of aerodynamic derivatives. G. Temple.

2075-A theoretical analysis of longitudinal dynamic stability in gliding flight. $H . M$.

Lyon, P. M. Truscott, E. I. Auterson and J. Whatham.

2101-Examples of the application of Busemann's formula to evaluate the aerodynamis force coefficients on supersonic aerofoils. C.N.H. Lock.

The following reports have also been received:-

Publications Scientifiques et Techniques du Ministère de l'Air.

Bulletins des Services Techniques. No. 109.

Notes Techniques. No. 24.

J. Laurence Pritchard,

Secretary. 

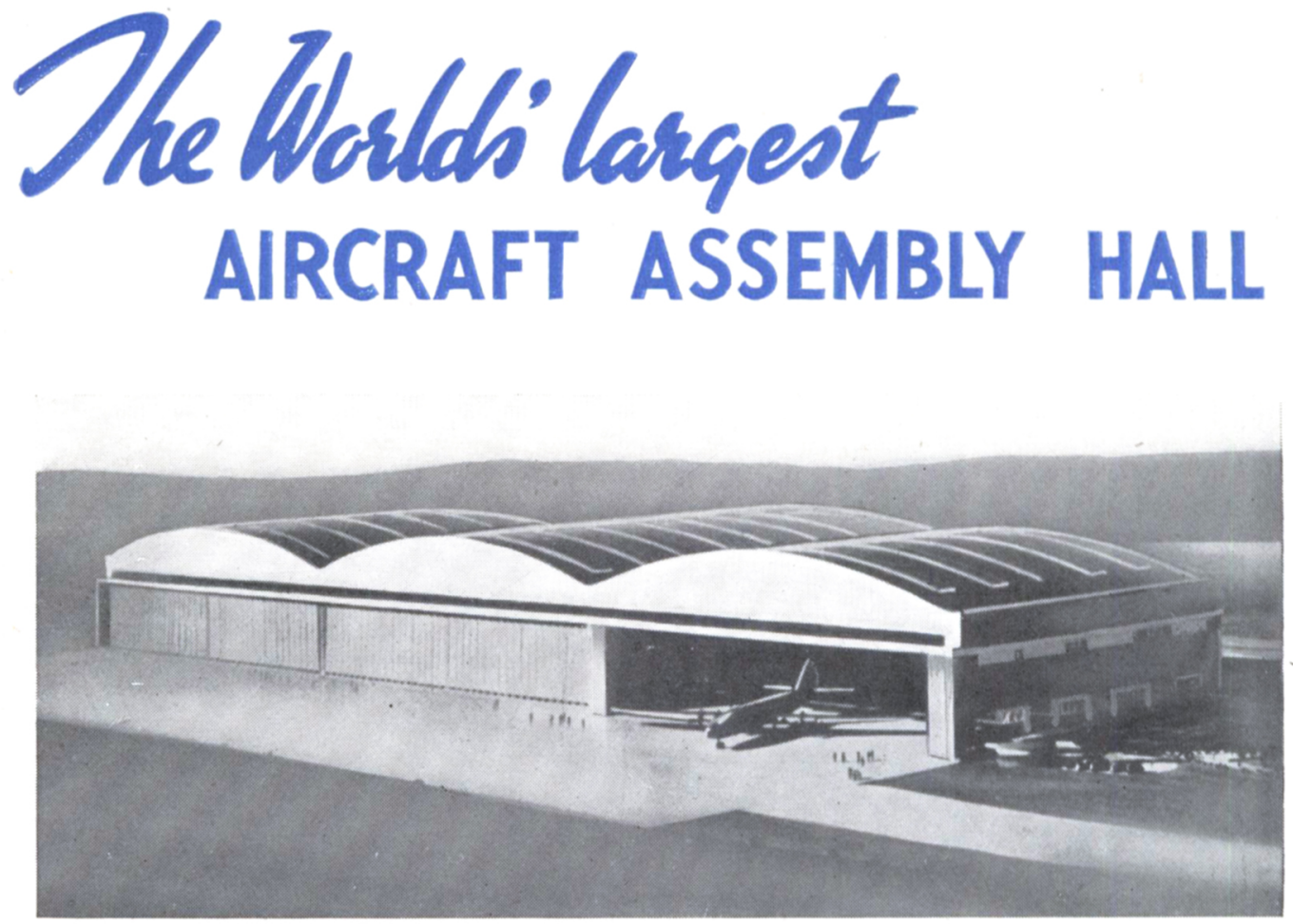

The largest aircraft assembly hall in the World is now being built at Filton for the erection of 125 ton giant "Bristol" Brabazon Aircraft. This immense structure, 1,052 feet wide and 420 feet deep, is wider than the length of the QUEEN MARY or the Houses of Parliament and covers $7 ! \frac{1}{2}$ acres. More than 5,000 yards of high and low tension cables for the complex electric power system and 450 main lighting groups are being supplied and installed by B.I.Callender's.

ARCHITECT : Eric Ross, A.R.I.B.A.

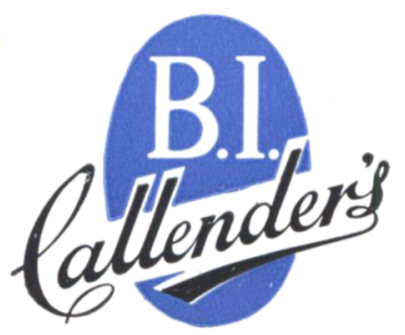

CONSULTING ENGINEERS: Brian H. Colquhoun \& Partners

BRITISH INSULATED CALLENDER'S CABLES LIMITED NORFOLK HOUSE. NORFOLK STREET, LONDON, W.C.2 


\section{Semice of Supply}

FOR JET ENGINES
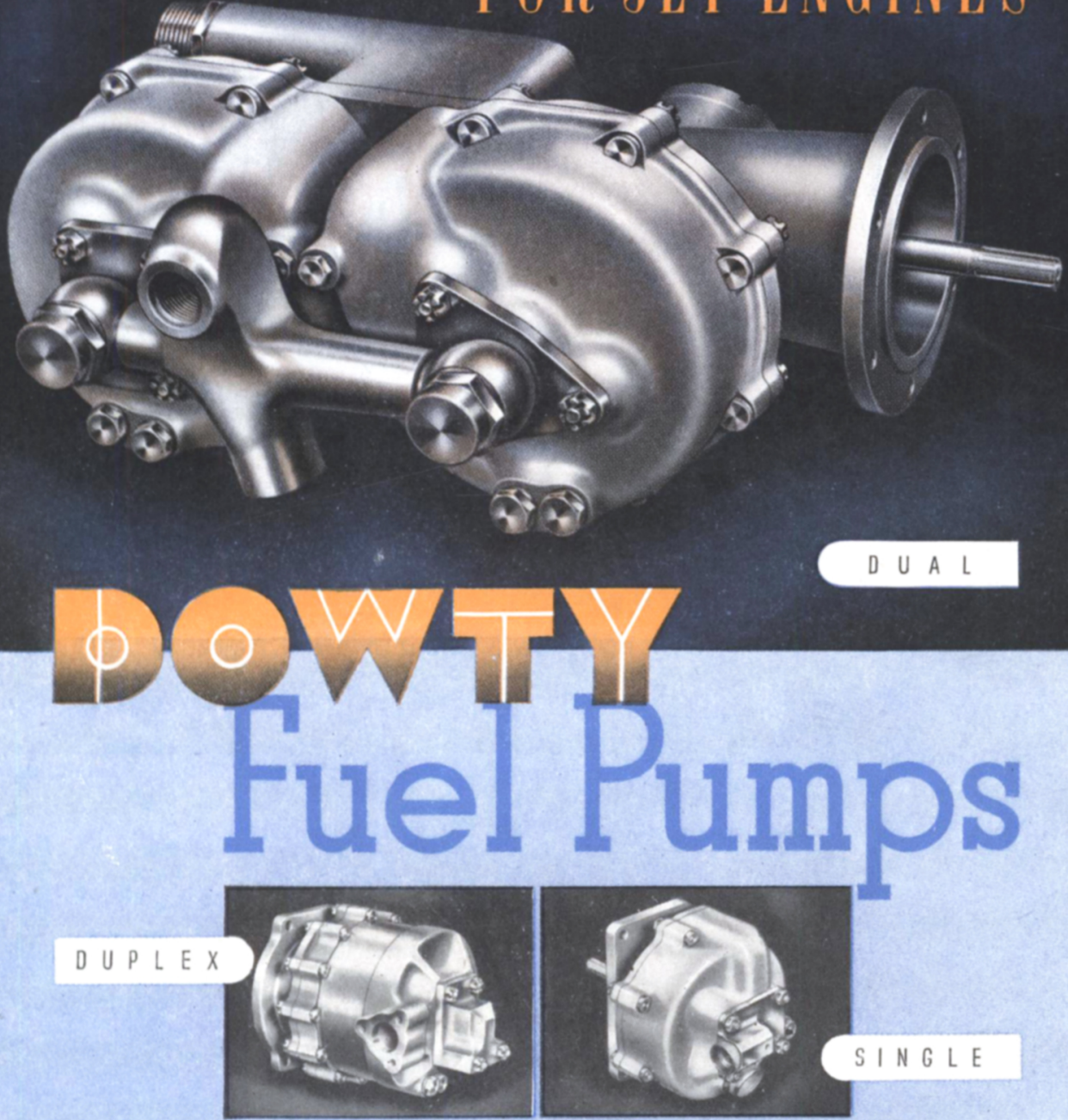

Dowty Fuel Pumps deliver 600 to 1,320 Imperial gallons per hour at 3.500 r.p.m. and 1,000 lbs. per square inch pressure

D O

Printed by the Lewes Press (Wightman \& Co. Ltd.), Friars Walk, I ewes, lingland, and Published by The Royal Aeronautical Society, 4 Hamilton Place, London, W.1, England. 